\title{
Evaluating the Social Acceptability of Voice Based Smartwatch Search
}

\author{
Christos Efthymiou, Martin Halvey \\ Department of Computer and Information Sciences, University of Strathclyde, Glasgow, UK \\ chefthimioulhotmail.com, martin.halveyestrath.ac.uk
}

\begin{abstract}
There has been a recent increase in the number of wearable (e.g. smartwatch, interactive glasses, etc.) devices available. Coupled with this there has been a surge in the number of searches that occur on mobile devices. Given these trends it is inevitable that search will become a part of wearable interaction. Given the form factor and display capabilities of wearables this will probably require a different type of search interaction to what is currently used in mobile search. This paper presents the results of a user study focusing on users' perceptions of the use of smartwatches for search. We pay particular attention to social acceptability of different search scenarios, focussing on input method, device form and information need. Our findings indicate that audience and location heavily influence whether people will perform a voice based search. The results will help search system developers to support search on smartwatches.
\end{abstract}

Keywords: Smartwatch, Acceptability, Voice, Search, Information Need

\section{Introduction}

Search using mobile devices is becoming more popular and this is set to continue as more devices with increased computing power become available. In addition, the range of devices people are using to access the web are increasing e.g. tablets, phones, smartwatches etc. As the range of devices increases the methods that people use to interact with these devices are also changing. In particular for mobile search this has resulted in the development of both voice based search systems e.g. Cortana, Siri etc. and proactive card based search systems [1], as opposed to traditional reactive search with ranked lists. A lot of effort and research has gone into developing both the hardware for these devices and also the intelligent software that allows voice based search for example. Much less research has looked into social factors surrounding these devices and their use i.e. how users will feel about using them for search on the move etc. Social acceptability issues have in part contributed to some high profile technology failures e.g. Google Glass. We want to address this lack of acknowledgment of social acceptability issue for smartwatches and in particular for search using smartwatches. Thus in this paper we investigate the social acceptability of using a smartwatch to search. In particular we focus on reactive search (i.e. search initiated by a

adfa, p. 1, 2011.

(C) Springer-Verlag Berlin Heidelberg 2011 
user) and in particular on querying using a smartwatch where input is predominantly voice based. Specifically we focus on the following research questions:

RQ1: Does information need determine when and where an individual would use a smartwatch for search?

RQ2: Does the form factor of the device determine when and where an individual would use a smartwatch for search?

RQ3: Does the input method determine when and where an individual would use a smartwatch for search?

RQ4: Does the expression of information need determine when and where an individual would use a smartwatch for search?

To address these research questions a lab based user study following a methodology proposed by Rico et al. [2] was conducted; videos depicting various interaction scenarios (in our case users searching on mobile devices i.e. phone and smartwatch) were presented and participant responses were elicited. The aim was to gather user perceptions of the social acceptability of the specific scenarios presented.

\section{Related Work}

\subsection{Mobile Search Behaviour}

Jones et al. [3] evaluated users' abilities on mobile phones, PDAs, and desktop interfaces for some of the earliest mobile search systems. They found that both the search speed and accuracy were worse on smaller screens. Church et al. [4] analysed almost 6 million individual search requests produced by over 260,000 individual mobile searchers over a 7 days period in 2006. At that time mobile search was only used frequently used by $8-10 \%$ of mobile internet users. Church et al. also noted that users had a limited set of information needs, with a high number of transactional and navigational queries ([5]) and a high number of adult/pornographic queries. A number of researchers have looked at social setting and how that influences mobile information needs. According to Kassab and Yuan [10] mobile users are motivated to use mobile phones to seek information motivated from conversations with other people, to view their emails and download mobile applications. Church et al. [6] found that the majority of mobile users, use mobile search to seek information about trivia and pop culture things. Furthermore, they found that the social mobile search is more likely to take place in an unfamiliar locations. As a result Church et al. [7] developed the Social Search Browser (SSB), an interface that embodies social networking abilities with important mobile contexts. Location also plays an important part in social context. Ren et al. [8] investigated how mobile users use the web in large indoor spaces, specifically retail malls, to look for information. Church and Cramer [9] have recently looked at the requirements of place in local search in purpose to improve the locationbased search in the future. Other researchers have looked at how search is changing as a result of device changes. Montanez et al. [10] have looked at the range of devices that people use to satisfy information needs, when particular devices are used and when people switch between these devices. As devices are evolving mobile search is beginning to move from being reactive into being more proactive. With this in mind 
some researchers have begun to look at card based retrieval and in particular how they are influenced by social situations [1]. Most wearable search systems operate a combination of reactive and proactive card based retrieval systems. This does not eliminate the need for reactive search and some sort of input e.g. systems like Siri and Cortana encourage voice input and a dialogue with the system.

\subsection{Social Acceptability}

Rico and Brewster [2] investigated the social acceptability of a set of gestures for interacting with mobile devices with respect to location and the audience. Within the area of wearables, Schaar and Ziefle [11] evaluated the acceptance of smart shirts in men and women and found that men were more accepting of the technology than women. Shinohara and Wobbrock [12] looked at the perception of a variety of users of assistive technology. Whilst this technology was extremely useful, some of participants felt stigmatised by their assistive technology. A study looking at the privacy behaviour of life loggers [13] found that for life loggers a combination of factors including time, location, and the objects and people appearing in a photo determines its 'sensitivity;' and that life loggers are concerned about the privacy of bystanders. Specifically for smartwatches, Pearson et al. [14] investigated the interaction and the process of attracting attention when glancing at someone else's watch. Bystanders not only could notice the information and content on someone else's smartwatch screen, but they were likely to interact with the wearer and tell them about information presented on their smartwatch screen. Moorthy and $\mathrm{Vu}$ [15] conducted an evaluation of the use of a Voice Activated Personal Assistant in the public space. Users were quite careful to reveal their private information in front of strangers and less careful with their non-private information. This behaviour difference was heightened in public locations and especially when obvious methods were used for information input.

\section{User Evaluation}

\subsection{Information Need Survey}

For our user evaluation we required a set of real mobile information needs, these were gathered through an online survey. For each information need we asked participants for a clear and precise single sentence that describes the information need (e.g. "What year Winston Churchill born?"). We then asked for a description of what constituted relevant information for this information need. We then asked participants to provide queries or keywords that they used to satisfy this information need. This follows the method used by Wakeling et al. for gathering information needs for their study of relevance of search results [16]. Finally, we asked what mobile device had been used to satisfy their information need (e.g. phone, tablet etc.) providing the make and the model of the mobile device. The survey had 83 respondents. 44 (53.7\%) were male, $38(46.3 \%)$ were female and 1 respondent declined to provide this information. Respondent's ages varied between $21-66$ years old, the average age was 30.61 . With respect to mobile search $11(13.3 \%)$ participants indicated they had low experience, 
$28(33.7 \%)$ had a medium level of experience and $44(53 \%)$ indicated that they have high experience. The mobile information needs provided were analysed. Similar to the information needs analysis by Church et al. [5] there were a large number of informational information needs $(79.4 \%)$, some transactional $(17.8 \%)$ and very little navigational (2.73\%). Initially the categories from Church et al. [5] were used for categorisation, but they did not appear to capture the information needs sufficiently well, so instead bottom up categorisation of information needs was created. One key difference between our work and that of Church et al. [5] is Church et al. used log analysis and our participants may be reluctant to outline some of their information needs e.g. Adult. This resulted in 11 categories, which were slightly different from those of Church et al., e.g. News and Weather featured in our categories, but Adult did not. Also News and Weather whilst common were not amongst the most popular categories. The three most popular categories were Directions, Entertainment and General Search (used interchangeably with Search with a capital S in parts of the paper), we use these in our user study. For reasons of space and as it is not the focus of the paper we do not go into detail about the categorisation. Our aim was to have representative information needs which could be used in our user study.

\subsection{Procedure}

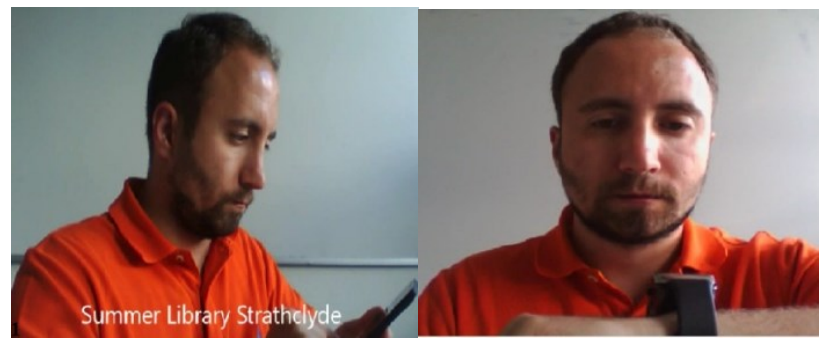

Fig. 1. Screenshots from example videos.

Table 1. Example queries and conditions, not all are shown for reasons of space.

\begin{tabular}{lllll}
\hline Query & Info Need & Device & Input Method & Expression \\
\hline 'What fireworks displays are being offered tonight?' & Entertainment & Phone & Voice & Statement \\
'I want to find data analytics articles' & Search & Phone & Text & Statement \\
'Glasgow Edinburgh bus times' & Directions & Watch & Voice & Keywords \\
\hline
\end{tabular}

We followed the procedure of Rico and Brewster [2], where participants watched a set of videos depicting interaction scenarios and were then asked to provide responses regarding the scenarios depicted in the video. A user evaluation in real locations was also considered, however that may require placing participants in potentially embarrassing and uncomfortable positions. In addition it would not allow us to consider as many locations and audiences as we can with this in lab study. In total there were 18 videos. Each video had an information depicted a person searching for information, each search had an information need (General Search, Directions, Entertainment) a query input (consisting of a device (Phone, Watch) and input method (Text, Voice) 
pair) and an expression type (Statement, Keyword). Information needs were based on the real information needs gathered in the online survey (see Section 3.1). Device allowed us to compare smartwatch and phone; mobile phones are the most common way for people to currently search whilst mobile. Input method allows comparison between the more common text entry and voice input. Three pairs were used, text entry on a smartwatch was omitted. Text entry on such a small screen is impractical and remains an area of open research [17]. Finally expression of the query could be a statement or in the form of keywords. For many voice based systems querying with statements is common e.g. to view your steps on an Android device the statement is "okay Google, show me my steps". Whereas for text based search keywords are more prevalent. Keywords were the keywords provided by respondents and the statements were the single sentence that we requested. Before beginning participants were told the aim of the study was to assess the social acceptability of different mobile searches.

For each search scenario participants watched a video of the search being performed and answered multiple-choice questions. The videos lasted between four and ten seconds each and participants answered the associated questions after each video, they could request a video to be replayed. Each video portrayed a search being performed by a male actor sitting in front of a plain background. Where voice was used as input audio was provided, when text was an input the query ran along the bottom of the screen. The devices used in the video were a Nexus 5 phone and a Samsung Gear Live smartwatch. Figure 1 shows frames from two of the videos. Because participants were asked to imagine the locations and audiences where they might perform these searches the videos were designed to focus solely on the search scenario itself. The videos used in this study intentionally portrayed a plain scene without a defined context so that the setting would not distract viewers from evaluating the search input. After watching each video, participants were asked to select from a list of the locations where they would be willing to perform the given search. Users were then asked to select from a list of the types of audiences they would be willing to perform the search in front of. These audiences and locations are based on work by Rico and Brewster [2]. There were six locations (Driving, Home, Passenger, Pavement, Pub/Restaurant, Workplace) and six audiences (Alone, Colleagues, Family, Friends, Partner, Strangers). These responses intentionally asking participants to imagine themselves in these settings in a first person rather than second person view [18] in order to focus on one's personal actions rather than opinions of other's. The order of video presentation was randomised. Overall this study had a total of 4 independent variables; information need, device type, input type and expression type. Example videos and combinations of variables are presented in Table 1. For each of these independent variables, 2 dependent variables (audience acceptance and location acceptance) were analysed. As the data gathered was found to not be normally distributed, non-parametric statistical tests were used, those being Friedman Tests and Wilcoxon Sign Rank Tests. For each variable presented below we first look at the impact of the variable itself (e.g. information need), we then compare each location and audience between variables (e.g. Entertainment in front of Friends vs. Directions in front of Friends) and finally we look at the location and audience for 
each instance of each variable (e.g. compare all audiences for directions information needs) following Rico and Brewster [2].

\subsection{Participants}

There were 20 participants in our user study with age range from 23 to 32 and average age 25.15. Participants were mostly recruited from the University of Strathclyde. All of the participants lived in the UK and spoke English, but were from a range of countries. $5(25 \%)$ of the participants were female and $15(75 \%)$ were male.

\section{$4 \quad$ Results}

\subsection{Information Need}

Table 2. Average acceptance rates for different Information Needs by location.

\begin{tabular}{lllllll}
\hline & Home & Driv. & Pub & Pave. & Pass. & Work \\
\hline Enter. & $88 \%$ & $31 \%$ & $32 \%$ & $53 \%$ & $40 \%$ & $22 \%$ \\
Search & $95 \%$ & $18 \%$ & $16 \%$ & $36 \%$ & $38 \%$ & $41 \%$ \\
Direc. & $94 \%$ & $38 \%$ & $28 \%$ & $63 \%$ & $58 \%$ & $33 \%$ \\
\hline
\end{tabular}

Table 3. Average acceptance rates for different Information Needs by audience.

\begin{tabular}{lllllll}
\hline & Alone & Fam. & Coll. & Part. & Frie. & Stran. \\
\hline Enter. & $93 \%$ & $73 \%$ & $23 \%$ & $81 \%$ & $88 \%$ & $19 \%$ \\
Search & $98 \%$ & $53 \%$ & $38 \%$ & $62 \%$ & $68 \%$ & $18 \%$ \\
Direc. & $99 \%$ & $84 \%$ & $38 \%$ & $90 \%$ & $93 \%$ & $42 \%$ \\
\hline
\end{tabular}

A Friedman Test revealed significance for information needs have significant effect on location $\left(\mathrm{X}^{2}(2)=27.966, \mathrm{p}<0.001\right)$ (see Table 2 ). Pairwise comparisons with a Wilcoxon Sign Rank test (Bonferroni adjusted alpha $\mathrm{p}=0.0167$ ) revealed significant differences between Directions and Entertainment $(\mathrm{z}=-3.495, \mathrm{p}<0.001)$ as well as Directions and Search $(\mathrm{z}=-5.215, \mathrm{p}<0.001)$. With respect to audience (see Table 3 ) there was also a significant difference for information need $\left(\mathrm{X}^{2}(2)=78.072, \mathrm{p}<0.001\right)$. Pairwise comparisons revealed a significant difference between all combinations, Search-Entertainment $(\mathrm{z}=-3.162, \mathrm{p}=0.002)$, Search-Directions $(\mathrm{z}=-8.255, \mathrm{p}<0.001)$, and Entertainment-Directions $(\mathrm{z}=-5.914, \mathrm{p}<0.001)$. Pairwise comparisons were made for each location and audience (Bonferroni adjusted alpha $\mathrm{p}=0.0014$ ). For location there were significant differences between Searching and Directions for Driving $(p=0.001)$, Pavement $(p=0.001)$ and Passenger $(p=0.001)$. As can been seen in Table 2 these are locations where participants reported that they would be likely to look for Directions but not conduct a General Search. There were a higher number of significant differences in terms of audience. As with location the highest number were between Searching and Directions where there were significant differences for Family $(p=0.001)$, Partner $(p=0.001)$, Friends $(p=0.001)$ and Strangers $(p=0.001)$. Between 
Searching and Entertainment there were differences in audience for Partner $(p=0.001)$ and Friends $(\mathrm{p}=0.001)$. Finally for Entertainment and Directions there was a difference for Strangers $(\mathrm{p}=0.001)$. Overall Directions was the most acceptable, followed by Entertainment and General Search being the least acceptable for all audiences.

Looking at each information need individually. For Entertainment, Home was the most acceptable search location (see Table 2) and is significantly more acceptable than any other location ( $\mathrm{p}<0.001$ for all comparisons). Pavement is a more acceptable location than Driving $(\mathrm{p}=0.001)$, Pub $(\mathrm{p}=0.001)$ or the Workplace $(\mathrm{p}<0.001)$. Workplace is also less acceptable than being a Passenger. With respect to Audience, searching for Entertainment related information is least acceptable in front of Strangers, which is significantly different to Alone $(p<0.001)$, Family $(p<0.001)$, Partner $(\mathrm{p}<0.001)$ or Friends $(\mathrm{p}<0.001)$. Searching Alone is also significantly different to in front of Colleagues $(p<0.001)$ or Family $(p<0.001)$, Alone is seen as the most acceptable audience (see Table 3). Colleagues as an audience is also significantly less acceptable than in front of Family $(p<0.001)$, Partners $(p<0.001)$ or Friends $(p<0.001)$. Family and Friends also has a significant difference $(p<0.001)$.

In terms of the Searching category there were significant differences between the acceptability of almost all locations. There was no significant difference for Workplace with Pavement $(\mathrm{p}=0.446)$ and Passenger $(\mathrm{p}=0.579)$. For Passenger there was no significant difference with Pavement $(\mathrm{p}=0.670)$. All of these locations were mid-range in terms of acceptability compared to other locations (see Table 2). Pub and Driving were not significantly different $(\mathrm{p}=0.732)$, both were seen as the least acceptable (see Table 2). Similarly for audience with respect to search most audiences had significantly different acceptance rates. Family had similar acceptance to Colleagues $(\mathrm{p}=0.024)$ and Partner $(\mathrm{p}=0.068)$. With Partner also having a similar acceptance rate to Friends $(\mathrm{p}=0.131)$.

With respect to Directions, the majority of locations had significantly different acceptance rates. Workplace had a similar acceptance level to Driving $(\mathrm{p}=0.327)$ and Pub ( $\mathrm{p}=0.355)$. Pub and Driving also had similar acceptance rates $(\mathrm{p}=0.085)$. All had low acceptance rates in comparison to other locations. Passenger and Pavement also had non-significant differences in terms of acceptance rate $(\mathrm{p}=0.257)$. In terms of audience, there were less significant differences for Directions, with relatively high acceptance rates. That being said the most acceptable audiences Alone was more acceptable than Family $(p<0.001)$, Colleagues $(p<0.001)$, Partner $(p=0.002)$ and Strangers $(p<0.001)$. Strangers the least acceptable audience was also significantly less acceptable than Family $(p<0.001)$, Partner $(p<0.001)$ and Friends $(p<0.001)$. Colleagues which was the second least acceptable audience and was significantly less acceptable than Partner $(p<0.001)$, Friends $(p<0.001)$ and Family $(p<0.001)$.

\subsection{Device}

The Device variable had a significant effect on location $(\mathrm{z}=-2.538, \mathrm{p}=0.011)$ and on audience $(\mathrm{z}=-2.121, \mathrm{p}=0.034)$, with the use of Phone being more acceptable on almost all cases, see Table 4 and Table 5. Individual pair-wise comparisons between Phone and Watch for every location and audience were also conducted using a Wilcoxon 
Sign Rank test (Bonferroni adjusted alpha $p=0.004$ ) and the only significant difference was for the Driving location $(\mathrm{z}=4.808, \mathrm{p}<0.001)$, with using a Watch whilst Driving being more acceptable than a Phone. Although not significant the use of Watch rather than Phone for searching was more acceptable in front of Family $(\mathrm{z}=2.252, \mathrm{p}=0.024)$. We also performed pairwise comparisons individually for both the Phone and Watch variables between every location and audience (Bonferroni adjusted alpha $\mathrm{p}=0.003$ ). For reasons of space we do not present all of the results but rather summarise them here. Looking at Phone first, there were significant differences between all locations with the exception of Driving-Pub $(\mathrm{p}=0.088)$, PavementPassenger $(p=1.0)$ and Workplace-Pub $(p=0.083)$. In terms of audience, again for Phone almost all audiences are significantly different, the exceptions being FriendsPartner $(p=0.02)$ and Colleagues-Strangers $(p=0.107)$. Next looking at pairwise comparisons within the Watch variable we see that for location that Driving is as acceptable as on Pavement $(p=0.317)$ or as a Passenger $(p=0.121)$ and that Workplace is as acceptable as Pub $(p=0.303)$ or Passenger $(p=0.063)$. Searching in front of Friends $(\mathrm{p}=0.059)$ and Partners $(\mathrm{p}=0.819)$ is as acceptable as Family, with Friends and Partners also being similarly acceptable $(\mathrm{p}=0.162)$. With searching in front of Strangers and Colleagues also being similarly acceptable $(\mathrm{p}=0.04)$

Table 4. Average acceptance rates for Phone versus Watch by location.

\begin{tabular}{lllllll}
\hline & Home & Driv. & Pub & Pave. & Pass. & Work \\
\hline Phone & $96 \%$ & $21 \%$ & $28 \%$ & $51 \%$ & $51 \%$ & $36 \%$ \\
Watch & $85 \%$ & $44 \%$ & $18 \%$ & $50 \%$ & $34 \%$ & $23 \%$ \\
\hline
\end{tabular}

Table 5. Average acceptance rates for Phone versus Watch by audience.

\begin{tabular}{lllllll}
\hline & Alone & Fam. & Coll. & Part. & Frie. & Stran. \\
\hline Phone & $97 \%$ & $68 \%$ & $36 \%$ & $79 \%$ & $84 \%$ & $30 \%$ \\
Watch & $95 \%$ & $74 \%$ & $28 \%$ & $75 \%$ & $81 \%$ & $18 \%$ \\
\hline
\end{tabular}

\subsection{Input Method}

In terms of Input Method there was no significant difference between Text and Voice for location $(\mathrm{z}=-1.472, \mathrm{p}=0.141)$, but there was for audience $(\mathrm{z}=-2.466, \mathrm{p}=0.014)$. Whilst overall location did not have an impact we see that for pairwise comparisons based on locations that there were significant differences (see Tables 6 and 8). With Text being more acceptable at Home, as a Passenger and in Work; whilst Voice input is deemed more acceptable whilst Driving. In terms of audience, using Text input is significantly more acceptable in front of Strangers and Colleagues (see Tables 7 and 8). Looking at pairwise comparisons for Text input per location (Bonferroni adjusted alpha $\mathrm{p}=0.003$ ), we found that Workplace is not significantly different to Pub $(p=0.01)$, Pavement $(p=0.325)$ and Passenger $(p=0.013)$. With Passenger and Pavement also not differing significantly $(p=0.048)$. With respect to audience we see no significant different between Partner and Friends $(p=0.007)$ and Family $(p=0.016)$, as 
well as no difference between Strangers and Colleagues $(\mathrm{p}=0.752)$. Looking at Voice input, between locations there are no significant differences for Driving with Pavement $(\mathrm{p}=0.03)$ and Passenger (0.514), and also between Pub and Workplace $(\mathrm{p}=0.659)$. As for audience, Partner has no significant difference with Family $(p=0.024)$ and Friends $(p=0.101)$, and there is no significant differences between Strangers and Colleagues $(\mathrm{p}=0.003)$.

Table 6. Average acceptance rates for Voice versus Text input by location.

\begin{tabular}{lllllll}
\hline & Home & Driv. & Pub & Pave. & Pass. & Work \\
\hline Text & $98 \%$ & $12 \%$ & $34 \%$ & $58 \%$ & $67 \%$ & $51 \%$ \\
Voice & $90 \%$ & $38 \%$ & $20 \%$ & $47 \%$ & $35 \%$ & $22 \%$ \\
\hline
\end{tabular}

Table 7. Average acceptance rates for Coice versus Text input by audience.

\begin{tabular}{lllllll}
\hline & Alone & Fam. & Coll. & Part. & Frie. & Stran. \\
\hline Text & $98 \%$ & $68 \%$ & $48 \%$ & $77 \%$ & $84 \%$ & $47 \%$ \\
Voice & $96 \%$ & $71 \%$ & $26 \%$ & $78 \%$ & $83 \%$ & $16 \%$ \\
\hline
\end{tabular}

Table 8. Pairwise comparison between Text and Voice for every location and audience. Significance value set using a Bonferroni correction at $\mathrm{p}=0.004$.

\begin{tabular}{llll}
\hline Location & $\mathrm{p}$ value & Audience & $\mathrm{p}$ value \\
\hline Home & $<0.001$ & Alone & 0.102 \\
Driving & $<0.001$ & Family & 0.302 \\
Pub & 0.123 & Colleagues & $<0.001$ \\
Pavement & 0.101 & Partner & 0.435 \\
Passenger & $<0.001$ & Friends & 0.262 \\
Workplace & $<0.001$ & Strangers & $<0.001$ \\
\hline
\end{tabular}

\subsection{Expression}

For Expression of information need there was no significant effect on location $(\mathrm{z}=-$ $1.362, \mathrm{p}=0.173)$, however, there was a significant difference for audience $(\mathrm{z}=-3.500$, $\mathrm{p}=0.001$ ). Pairwise comparisons between all audiences revealed that the only significant difference between Statements and Keywords was for Partner $(\mathrm{z}=-2.959$, $\mathrm{p}=0.003)$. Looking at Keywords and Statements separately. For location and Statement no significant difference was found between Workplace and Driving $(\mathrm{p}=0.655)$, Pub $(p=0.485)$ and Passenger $(\mathrm{p}=0.021)$; Passenger also had no significant difference with Driving $(\mathrm{p}=0.081)$ and Pavement $(\mathrm{p}=0.02)$; Pub and Driving $(\mathrm{p}=0.258)$ also had no significant difference. Audience seems to have more of an impact, with Partner having no significant difference to Family $(\mathrm{p}=0.006)$ and Friends $(\mathrm{p}=0.016)$; and no significant difference between Strangers and Colleagues $(\mathrm{p}=0.033)$. Looking at location for Keywords we found a similar pattern to with Workplace not being significantly different to Driving $(\mathrm{p}=0.127)$ or Pub $(\mathrm{p}=0.029)$; Passenger and Pavement are not significantly different $(\mathrm{p}=0.763)$; Driving and Pub are not significantly different 
$(p=0.642)$. In terms of audience, for Keywords, there is no significant difference for Partner with Family $(p=0.071)$ and Friends $(p=0.201)$; as well as no significant difference between Strangers and Colleagues $(p=0.170)$.

Table 9. Average acceptance rates for Statements versus Keywords by location.

\begin{tabular}{lllllll}
\hline & Home & Driv. & Pub & Pave. & Pass. & Work \\
\hline State. & $91 \%$ & $31 \%$ & $26 \%$ & $50 \%$ & $41 \%$ & $29 \%$ \\
Key. & $93 \%$ & $27 \%$ & $24 \%$ & $51 \%$ & $50 \%$ & $34 \%$ \\
\hline
\end{tabular}

Table 10. Average acceptance rates for Statements versus Keywords by audience.

\begin{tabular}{lllllll}
\hline & Alone & Fam. & Coll. & Part. & Frie. & Stran. \\
\hline State. & $96 \%$ & $64 \%$ & $34 \%$ & $73 \%$ & $81 \%$ & $25 \%$ \\
Key. & $97 \%$ & $76 \%$ & $33 \%$ & $82 \%$ & $86 \%$ & $28 \%$ \\
\hline
\end{tabular}

\section{DISCUSSION}

Information Need (RQ1): Analysis of the information needs provided in our online survey revealed that as Church found [5] there are a large number of informational mobile queries. The three most common categories from our analysis of mobile information needs were used for comparison in our in lab user study; those being Entertainment, Directions and General Search. There was a significant difference in terms of acceptability between all of the information needs. Directions, Entertainment and General Search were all viewed as being most to least acceptable in order. These information needs can be viewed as having a temporal aspect (Directions having an immediate temporal aspect). Also Directions and then Entertainment could be viewed as being easily displayed on the small screen of a smartwatch. In terms of audience questionnaire responses indicated that people are comfortable searching Alone. When they search in front of others they would rather do so in front of familiar audiences like Friends or Family, with searching for any information need in front of Strangers seen as being unacceptable. Some of the reasons for this are highlighted in the post evaluation interview, with issues of appearing strange and also privacy being highlighted as major concerns. With respect to location of performing a search again there are major differences, locations where a person might be alone or not actively engaged in other activities (i.e. Passenger and Pavement) are most acceptable for all information needs. The Workplace location has a different distribution to other locations, in that it is the only location where General Search is seen as being most acceptable in comparison to other locations.

Form Factor (RQ2): In our study device was seen to have a significant impact on the acceptability with respect to both audience and location. In general when directly comparing locations and audiences Phone was more acceptable than Watch, the exception being that search via smartwatch was significantly more acceptable when Driving. The trend and comparison when looking at pairwise comparisons for both Phone and Watch had similar distributions of acceptance. As a smartwatch is a rela- 
tively new technology this difference in acceptability may even out as smartwatches become more mainstream.

Input Method (RQ3): In our study location did not have a significant effect on acceptability. However pairwise comparisons revealed a difference between Home, Driving, Passenger and Workplace; with Text being more acceptable in all those locations except Driving. There was significant effect of audience in terms of acceptability. For Colleagues and Strangers Text was significantly more acceptable than Voice. One interesting thing to note was that for Partner, Family and Friend audience's Voice was slightly more acceptable than Text. The audiences that are familiar to the participants seem to be more acceptable for Voice, whereas those with unfamiliar people Voice becomes a more unacceptable input method.

Expression (RQ4): In general Keywords are more acceptable than Statements. However as with Input Method, location did not have a significant effect on the acceptability of using Statements or Keywords. In contrast Audience did have a significant effect. The only significant pairwise difference was for the Partner audience, with Keywords being more acceptable than Statements. Overall expression appears to have less of an impact than any of the other variables that we investigated.

\section{Conclusion}

Wearable technology and also voice based search are relatively new technologies for many people. These technologies open up new possibilities for search interaction whilst mobile. In comparison with more traditional desktop based search this also creates a range of new possibilities, but also factors that must be taken into account, for designing search interactions. In this paper we have focussed on social acceptability issues surrounding using voice based reactive search with smartwatches and also mobile phones. We conducted a lab study where we presented participants with various search scenarios and solicited responses to the acceptability of those scenarios. Overall these findings explain some of the reluctance for search to move beyond text input on mobile phones. This also validates some of the move towards card based proactive search, where the device displays "results" without a query. Our findings also demonstrate that there are some cases/locations/audiences where different ways of searching might be preferable. It is not yet possible to completely remove proactive search with user input, thus it is important to understand all of the factors that influence search interaction. This work is a first step in that direction and the results here provide some guidance on the types of information needs and scenarios that require proactive search and those that might be better served by reactive. A combination of both may ultimately provide the best user experience.

\section{$7 \quad$ References}

1. Shokouhi, M., Guo, Q.: From Queries to Cards: Re-ranking Proactive Card Recommendations Based on Reactive Search History. 38th International ACM SIGIR Conference on Research and Development in Information Retrieval, pp. 695-704. (2015) 
2. Rico, J., Brewster, S.: Usable gestures for mobile interfaces: evaluating social acceptability. 28th ACM Conference Conference on Human Factors in Computing Systems, pp. 887-896. (2010)

3. Jones, M., Buchanan, G., Thimbleby, H.: Improving web search on small screen devices. Interacting with Computers 15, 479-495 (2003)

4. Church, K., Smyth, B., Bradley, K., Cotter, P.: A large scale study of European mobile search behaviour. 10th international conference on Human computer interaction with mobile devices and services, pp. 13-22. (2008)

5. Broder, A.: A taxonomy of web search. ACM Sigir forum, pp. 3-10. ACM, (2002)

6. Church, K., Cousin, A., Oliver, N.: I wanted to settle a bet!: understanding why and how people use mobile search in social settings. 14th international conference on Humancomputer interaction with mobile devices and services, pp. 393-402. (2012)

7. Church, K., Neumann, J., Cherubini, M., Oliver, N.: SocialSearchBrowser: a novel mobile search and information discovery tool. In: Proceedings of the 15 th international conference on Intelligent user interfaces, pp. 101-110. (2012)

8. Ren, Y., Tomko, M., Ong, K., Sanderson, M.: How people use the web in large indoor spaces. 23rd ACM International Conference on Conference on Information and Knowledge Management, pp. 1879-1882. (2014)

9. Church, K., Cramer, H.: Understanding Requirements of Place in Local Search. 33rd Annual ACM Conference Extended Abstracts on Human Factors in Computing Systems, pp. 1857-1862. (2015)

10. Montañez, G.D., White, R.W., Huang, X.: Cross-device search. 23rd ACM Conference on Conference on Information and Knowledge Management, pp. 1669-1678. (2015)

11. Schaar, A.K., Ziefle, M.: Smart clothing: Perceived benefits vs. perceived fears. 5th IEEE Pervasive Computing Technologies for Healthcare, pp. 601-608. (2011)

12. Shinohara, K., Wobbrock, J.O.: In the shadow of misperception: assistive technology use and social interactions. 29th ACM Conference Conference on Human Factors in Computing Systems, pp. 705-714. (2011)

13. Hoyle, R., Templeman, R., Armes, S., Anthony, D., Crandall, D., Kapadia, A.: Privacy behaviors of lifeloggers using wearable cameras. ACM International Joint Conference on Pervasive and Ubiquitous Computing, pp. 571-582. (2014)

14. Pearson, J., Robinson, S., Jones, M.: It's About Time: Smartwatches as Public Displays. 33rd ACM Conference Conference on Human Factors in Computing Systems, pp. 12571266. (2015)

15. Easwara Moorthy, A., Vu, K.-P.L.: Privacy Concerns for Use of Voice Activated Personal Assistant in the Public Space. International Journal of Human-Computer Interaction 31, 307-335 (2015)

16. Wakeling, S., Halvey, M., Villa, R., Hasler, L.: A Comparison of Primary and Secondary Relevance Judgements for Real-Life Topics. ACM SIGIR Conference on Human Information Interaction and Retrieval, pp. 173-182. (2016)

17. Leiva, L.A., Sahami, A., Catalá, A., Henze, N., Schmidt, A.: Text Entry on Tiny QWERTY Soft Keyboards. 33rd Annual ACM Conference on Human Factors in Computing Systems, pp. 669-678. (2015)

18. Montero, C.S., Alexander, J., Marshall, M.T., Subramanian, S.: Would you do that?: understanding social acceptance of gestural interfaces. 12th international conference on Human computer interaction with mobile devices and services, pp. 275-278. (2010) 